\title{
Rational Design for Enhanced Acyltransferase Activity in Water Catalyzed by the Pyrobaculum calidifontis VA1 Esterase
}

\author{
Amanda Staudt ${ }^{1,2}$, Henrik Terholsen ${ }^{1}{ }^{\mathbb{D}}$, Jasmin Kaur ${ }^{1}$, Henrik Müller ${ }^{1}$, Simon P. Godehard ${ }^{1}$, \\ Ivaldo Itabaiana Jr. ${ }^{3}$, Ivana C. R. Leal ${ }^{2}$ and Uwe T. Bornscheuer ${ }^{1, *(D)}$ \\ 1 Department of Biotechnology \& Enzyme Catalysis, Institute of Biochemistry, University of Greifswald, \\ 17487 Greifswald, Germany; amanda_staudt@hotmail.com (A.S.); henrik.terholsen@uni-greifswald.de (H.T.); \\ jasmin.kaur@stud.uni-greifswald.de (J.K.); henrikmuller1301@gmail.com (H.M.); \\ simon_godehard@icloud.com (S.P.G.) \\ 2 Laboratory of Natural Products and Biological Assays, Department of Natural Products and Food, Faculty of \\ Pharmacy, Federal University of Rio de Janeiro, Rio de Janeiro 21941-902, Brazil; ivanafarma@yahoo.com.br \\ 3 Laboratory of Technological Biochemistry and Biocatalysis, Department of Biochemical Engineering, School \\ of Chemistry, Federal University of Rio de Janeiro, Rio de Janeiro 21941-909, Brazil; ivaldoufrj@gmail.com \\ * Correspondence: uwe.bornscheuer@uni-greifswald.de
}

Citation: Staudt, A.; Terholsen, H.; Kaur, J.; Müller, H.; Godehard, S.P.; Itabaiana, I., Jr.; Leal, I.C.R.;

Bornscheuer, U.T. Rational Design for Enhanced Acyltransferase Activity in Water Catalyzed by the Pyrobaculum calidifontis VA1 Esterase. Microorganisms 2021, 9, 1790. https://doi.org/10.3390/ microorganisms 9081790

Academic Editors: Dietmar Haltrich and Daniel Kracher

Received: 26 July 2021

Accepted: 20 August 2021

Published: 23 August 2021

Publisher's Note: MDPI stays neutral with regard to jurisdictional claims in published maps and institutional affiliations.

Copyright: (c) 2021 by the authors. Licensee MDPI, Basel, Switzerland. This article is an open access article distributed under the terms and conditions of the Creative Commons Attribution (CC BY) license (https:// creativecommons.org/licenses/by/ $4.0 /)$.

\begin{abstract}
Biocatalytic transesterification is commonly carried out employing lipases in anhydrous organic solvents since hydrolases usually prefer hydrolysis over acyl transfer in bulk water. However, some promiscuous acyltransferases can catalyze acylation in an aqueous solution. In this study, a rational design was performed to enhance the acyltransferase selectivity and substrate scope of the Pyrobaculum calidifontis VA1 esterase (PestE). PestE wild type and variants were applied for the acylation of monoterpene alcohols. The mutant PestE_I208A is selective for (-)-menthyl acetate $($ E-Value $=55)$. Highly active acyltransferases were designed, allowing for complete conversion of (-)-citronellol to citronellyl acetate. Additionally, carvacrol was acetylated but with lower conversions. To the best of our knowledge, this is the first example of the biocatalytic acylation of a phenolic alcohol in bulk water. In addition, a high citronellol conversion of $92 \%$ was achieved with the more environmentally friendly and inexpensive acyl donor ethyl acetate using PestE_N288F as a catalyst. PestE_N288F exhibits good acyl transfer activity in an aqueous medium and low hydrolysis activity at the same time. Thus, our study demonstrates an alternative synthetic strategy for acylation of compounds without organic solvents.
\end{abstract}

Keywords: PestE; acyltransferase; protein engineering; biocatalysis; acyl transfer; transesterification; monoterpene acylation

\section{Introduction}

Hydrolytic enzymes are versatile biocatalysts with many industrial applications, due to their broad substrate scope, absence of cofactor requirements, stability in organic solvents, and good chemo-, regio-, and stereoselectivity [1-3]. Usually, these enzymes are applied for the hydrolysis of esters, amides, or lipids in aqueous systems, since water acts as a nucleophile in the reaction [4,5]. Still, these enzymes can also act as biocatalysts for condensation reactions and in alcoholysis [1].

The capacity of some hydrolases, especially lipases, to synthesize esters and amides under anhydrous conditions increases the applicability of these enzymes $[1,6,7]$. Nevertheless, hydrolases typically favor a hydrolytic reaction over acyl transfer in water [8-11], limiting the direct acylation of interesting substances found naturally in aqueous raw materials.

In contrast to conventional hydrolases, some promiscuous hydrolases/acyltransferases are able to catalyze acyl transfer in aqueous systems, presenting an opportunity for cascade reactions in aqueous solutions. This is also more environmentally friendly than ionic 
liquids [12] or often hazardous and expensive organic solvents [5,13], which are then no longer required. Examples of promiscuous acyltransferases are enzymes from the CAL-A superfamily [14], an aryl esterase from Mycobacterium smegmatis (MsAcT) [13,15,16], family VIII carboxylesterases [17], and the enzymes from the bacterial hormone-sensitive lipase (bHSL) family $[5,18]$.

Due to their acyltransferase activity in water, a lipase from Sphingomonas sp. (SpL) and the lipase A from Pseudozyma antarctica (CAL-A) were used for amide and ester synthesis in the presence of $4 \%$ and $50 \%$ water, respectively $[19,20]$. Regarding cascade reactions, the acyltransferase MsAcT was applied in a combination with a transaminase to synthesize $\mathrm{N}$-benzylacetamide from benzaldehyde [21]. Furthermore, MsAcT was investigated in our group, and we observed approximately $50 \%$ conversion in the synthesis of benzyl acetate in a transesterification reaction between vinyl acetate and benzyl alcohol at an equimolar ratio in an aqueous environment [13].

Recently, our group also discovered esterase Est8 as a promiscuous hydrolase/acyltransferase. Est8 was the first enzyme from the bacterial hormone-sensitive lipase (bHSL) family, for which promiscuous acyltransferase activity was described [18]. Subsequently, we developed a sequence-based prediction method for acyltransferase activity, demonstrating that the active site hydrophobicity is directly related to the acyltransferase activity towards non-polar acyl acceptors. This analysis made it possible to identify and biochemically characterize five bHSLs with high acyltransferase activity [5].

One of the most promising acyltransferases identified in the study by Müller et al. [5] was the Pyrobaculum calidifontis VA1 esterase (PestE). This carboxylesterase (PDB code: $3 Z W Q)$, first reported by Hotta et al. [22], is a highly thermostable biocatalyst. It has been shown to catalyze the hydrolysis of bulky substrates [22,23] and notably presents activity towards tertiary alcohols in transesterification reactions in organic solvents [6], which was associated with the high hydrophobicity inside the substrate-binding pocket [5,24]. Recognizing the high potential of this enzyme, in this work, we performed a rational design to enhance its acyltransferase activity for the biocatalytic synthesis of monoterpene esters in an aqueous solution without organic solvents.

\section{Materials and Methods}

\subsection{Material}

$( \pm)$-Linalool $(97 \%),( \pm)$-menthol $(\geq 98.0 \%),( \pm)$-citronellol (analytical standard), carvacrol (99\%), (-)-menthol (99\%), and vinyl acetate were purchased from Sigma-Aldrich. All other chemicals and solvents were purchased from Sigma, VWR, or Carl Roth. The synthetic gene encoding PestE for expression in Escherichia coli-subcloned into the pET-21a vector-was based on the gene reported by Hotta et al. [22].

\subsection{Gene Expression and Protein Purification}

Chemically competent $E$. coli BL21(DE3) cells were transformed with expression vectors by heat shock followed by cooling and then plated on LB agar containing $50 \mu \mathrm{g} \cdot \mathrm{mL}^{-1}$ ampicillin. Pre-cultures (4 mL LB containing $50 \mu \mathrm{g} \cdot \mathrm{mL}^{-1}$ ampicillin) were inoculated with single colonies and incubated overnight $\left(37^{\circ} \mathrm{C}, 180 \mathrm{rpm}\right)$. LB medium $(200 \mathrm{~mL}$ containing $50 \mu \mathrm{g} \cdot \mathrm{mL}^{-1}$ ampicillin) was inoculated with $0.1 \%(v / v)$ of the pre-culture and incubated $\left(37^{\circ} \mathrm{C}, 180 \mathrm{rpm}\right)$ until it reached an $\mathrm{OD}_{600}$ of 0.6 . Protein expression was induced by the addition of isopropyl- $\beta$-D-thiogalactopyranoside (IPTG) to a final concentration of $0.4 \mathrm{mM}$ followed by incubation for $\sim 20 \mathrm{~h}$ at $20^{\circ} \mathrm{C}$ at $180 \mathrm{rpm}$.

Cells were harvested by centrifugation at $4000 \times g$ and $4{ }^{\circ} \mathrm{C}$ for $15 \mathrm{~min}$, and the cell pellets were resuspended with $4 \mathrm{~mL}$ equilibration buffer $(50 \mathrm{mM}$ potassium phosphate, $300 \mathrm{mM}$ sodium chloride, $10 \mathrm{mM}$ imidazole, $\mathrm{pH}$ 8.0). Cells were disrupted by sonication on ice (two cycles of $5 \mathrm{~min}$ sonication (30\% intensity, $50 \%$ pulsed cycle)) using a SONOPULS HD 2070 (BANDELIN Electronic GmbH \& Co. KG, Berlin, Germany), and the lysates were clarified by centrifugation at $10,000 \times g$ and $4{ }^{\circ} \mathrm{C}$ for $30 \mathrm{~min}$. For purification, the crude lysates were applied to $1.5 \mathrm{~mL}$ Roti ${ }^{\circledR}$ Garose-His/Ni Beads (Carl Roth, Karlsruhe, Germany). 
The resins were washed with $15 \mathrm{~mL}$ washing buffer $(50 \mathrm{mM}$ sodium phosphate, $300 \mathrm{mM}$ sodium chloride, $20 \mathrm{mM}$ imidazole, $\mathrm{pH}$ 8.0) before target proteins were eluted with elution buffer (50 mM sodium phosphate, $300 \mathrm{mM}$ sodium chloride, $250 \mathrm{mM}$ imidazole, $\mathrm{pH} 8.0$ ). Elution fractions were treated at $80^{\circ} \mathrm{C}$ for $20 \mathrm{~min}(500 \mathrm{rpm})$, centrifuged $\left(17,000 \times g, 4{ }^{\circ} \mathrm{C}\right.$ for $5 \mathrm{~min}$ ), and the supernatant transferred to the storage buffer (50 $\mathrm{mM}$ potassium phosphate, $300 \mathrm{mM}$ sodium chloride, $\mathrm{pH}$ 8.0) using PD-10 desalting columns (GE Healthcare, Chalfont St Giles, UK). Protein concentrations were determined at $280 \mathrm{~nm}$ using a NanoDrop ${ }^{\mathrm{TM}}$ 1000 spectrophotometer (ThermoFisher, Darmstadt, Germany), while the purity of the proteins was investigated by SDS-PAGE. The purified enzymes were mixed with $0.1 \%(v / v)$ Triton-X-100 for storage at $4{ }^{\circ} \mathrm{C}$.

\subsection{In Silico Methods}

Structural analysis and molecular modeling experiments of the structure of PestE (PDB entry: 3ZWQ) were performed using YASARA (Vienna, Austria) [25] and UCSF Chimera (San Francisco, CA, USA) [26]. The substrate-binding sites in the PestE crystal structure were identified and analyzed using the VINA docking tool [27] of the YASARA software. Molecular modeling was performed by two different approaches: the first was by evaluating the residues responsible for the water network and changing the structure to minimize hydrolase activity. The second was by increasing the hydrophobicity using the Chimera software with visualization of hydrophobic and hydrophilic regions.

\subsection{Site-Directed Mutagenesis}

Variants were constructed using the Q $5^{\circledR}$ Site-Directed Mutagenesis Kit (New England Biolabs GmbH, Ipswich, UK). Non-overlapping DNA-oligonucleotides were designed using the online NEBaseChanger tool for the mutations: H95A: forward primer $\left(5^{\prime} \rightarrow 3^{\prime}\right)$ : CGTGGAGACTgcgGACCACGTGTGTAGGC; reverse primer $\left(5^{\prime} \rightarrow 3^{\prime}\right)$ : CTCCCCAA GACGAAGCCC; I208A: forward primer $\left(5^{\prime} \rightarrow 3^{\prime}\right)$ : CGAATACGTCgcg CTCACCGCCGACTTAATGG; reverse primer $\left(5^{\prime} \rightarrow 3^{\prime}\right)$ : GGCCCGCTGTACTCCACT; N288F: forward primer $\left(5^{\prime} \rightarrow 3^{\prime}\right)$ : CGGCTTCGTCtttTTCTACCCCATATTAGAAG, reverse primer $\left(5^{\prime} \rightarrow 3^{\prime}\right)$ : TGGATGACGCCGTTGTAC. PCR amplification and KLD reactions were performed according to the manufacturer's protocol. The correct introduction of the desired mutations was confirmed by sequencing by Eurofins Genomics GmbH (Ebersberg, Germany).

\subsection{SDS-PAGE Analysis}

The protein purity was analyzed by SDS-PAGE. Purified proteins were denatured by heating $\left(95^{\circ} \mathrm{C}, 10 \mathrm{~min}\right)$ in a $10 \%(\mathrm{w} / \mathrm{v})$ sodium dodecyl sulfate (SDS) solution followed by centrifugation at $13,000 \times g$ for $5 \mathrm{~min}$. The proteins were separated on $12.5 \%$ acrylamide gels at a constant voltage of $200 \mathrm{~V}$. A protein standard was added for protein size comparison (Pierce $^{\mathrm{TM}}$ Unstained Protein MW Marker, ThermoFisher, Darmstadt, Germany) and stained by Coomassie Brilliant Blue G-250.

\subsection{Biocatalytic Experiments}

Purified PestEs (wild type and variants) were applied for transesterification of linalool, menthol, carvacrol, and citronellol. For the reactions, a mixture of $20 \mathrm{mM}$ monoterpene (from a $1 \mathrm{M}$ stock in acetonitrile), vinyl acetate at a molar ratio of 1:10 (monoterpene:acyl donor), $0.1 \%(v / v)$ Triton-X-100 (from a $10 \%(v / v)$ stock in water), and $0.2 \mu \mathrm{g} \cdot \mathrm{mL}^{-1}$ of the purified PestE variants were adjusted to a total volume of $1 \mathrm{~mL}$ with buffer ( $50 \mathrm{mM}$ potassium phosphate, $300 \mathrm{mM}$ sodium chloride, $\mathrm{pH}$ 8.0). Reactions were performed in $1.5 \mathrm{~mL}$ reaction tubes and incubated at $40{ }^{\circ} \mathrm{C}$ and $1000 \mathrm{rpm}$ in a ThermoMixer Comfort (Eppendorf AG, Hamburg, Germany). Reactions without enzymes were performed to determine background transesterification and served as control. Time samples $(10 \mu \mathrm{L})$ were taken, quenched with $10 \mu \mathrm{L}$ of $2 \mathrm{M} \mathrm{HCl}$, and extracted with $200 \mu \mathrm{L}$ ethyl acetate (EtOAc). After drying with anhydrous $\mathrm{MgSO}_{4}$, samples were analyzed by GC. In order to evaluate the possibility of producing monoterpene esters with a more environmentally friendly and 
cheaper acyl donor, reactions were also performed for $( \pm)$-citronellol with ethyl acetate as acyl donor. The reaction conditions were described above, with $20 \mu \mathrm{g} \cdot \mathrm{mL}^{-1}$ of the purified PestE variants.

\subsection{GC Analysis}

Reactions with linalool, carvacrol, and citronellol were analyzed by GC-MS using a GCQP2010 SE (Shimadzu, Kyoto, Japan) equipped with a ZB-5MSi column $(30.0 \mathrm{~m} \times 0.25 \mathrm{~mm}$, $0.25 \mu \mathrm{m}$ film thickness, Phenomenex, Torrance, CA, USA). Injector temperature was $220^{\circ} \mathrm{C}$, a flow rate of $1.20 \mathrm{~mL} \cdot \mathrm{min}^{-1}$ was used, and $1 \mu \mathrm{L}$ sample at a split ratio of 10 was injected. The column temperature was held at $80^{\circ} \mathrm{C}$ for $3 \mathrm{~min}$, increased to $260^{\circ} \mathrm{C}$ at $10^{\circ} \mathrm{C} \cdot \mathrm{min}^{-1}$, totalizing a $21 \mathrm{~min}$ method. Mass spectrum ion source and interface temperature was $220^{\circ} \mathrm{C}$, and the identification started after $3 \mathrm{~min}$ of the run.

Biocatalysis reactions with menthol were analyzed by GC-MS using a GC-QP2010 SE (Shimadzu, Kyoto, Japan) equipped with a $\beta$-TBDAc column $(25.0 \mathrm{~m} \times 0.25 \mathrm{~mm}$, MachereyNagel, Düren, Germany). Injector temperature was $220^{\circ} \mathrm{C}$, a flow rate of $2.06 \mathrm{~mL} \cdot \mathrm{min}^{-1}$ was used, and $1 \mu \mathrm{L}$ sample at a split ratio of 30 was injected. The column temperature was held at $80^{\circ} \mathrm{C}$ for $5 \mathrm{~min}$, increased to $145^{\circ} \mathrm{C}$ at $1.75^{\circ} \mathrm{C} \cdot \mathrm{min}^{-1}$, held for $5 \mathrm{~min}$, and then increased to $180{ }^{\circ} \mathrm{C}$ at $20^{\circ} \mathrm{C} \cdot \mathrm{min}^{-1}$ and kept for $6.11 \mathrm{~min}$, resulting in a $55 \mathrm{~min}$ method. Mass spectrum ion source and interface temperature were $220^{\circ} \mathrm{C}$.

\section{Results}

An initial study regarding menthol acylation with known hydrolases/acyltransferases was performed in order to verify potential acyltransferases for monoterpene ester synthesis. For this, the enzymes PestE, 1EVQ (Est2 from Alicyclobacillus acidocaldarius), CAL-A (lipase A from Pseudozyma antarctica), and PLE-6 (Pig Liver Esterase 6) were applied as catalysts. PestE showed the highest activity in menthol acylation (Figure S1), achieving $81 \%$ of the esterified product but with undesired subsequent hydrolysis. This behavior is known as an obstacle for biocatalytic ester production in water, in view that the optimum time point for the highest conversion needs to be determined to stop the reaction immediately; this makes enzymatic acyl transfer reactions in water somewhat challenging [5]. In addition, the possibility of enabling enantioselective acylation of $( \pm)$-menthol by mutagenesis has been investigated.

Aiming to decrease product hydrolysis, PestE was used as an object for the rational design. To identify target residues for site-directed mutagenesis, molecular docking was performed using (-)-menthol as a model substrate to study its binding in the PestE active site, as shown in Figure 1.

Residues composing the substrate binding of (-)-menthol in the active site of PestE were examined. Three residues were selected, His95, Ile208, and Asn288, to perform rational protein engineering aiming to increase active site hydrophobicity and/or tunnel size. To enhance the hydrophobicity, the residues His 95 and Asn288 were selected, while Ile208 was selected to increase the tunnel size. The increase in the active site region hydrophobicity can promote a more favorable surrounding for organic nucleophiles than for water. Moreover, the tunnel is the cavity space that connects the protein surface to the active site, and the residues forming this tunnel can have a significant influence on the biocatalytic properties so that the decrease in the residue size in these positions can increase the acceptance of larger substrates and the flux of substrates and products [28]. 


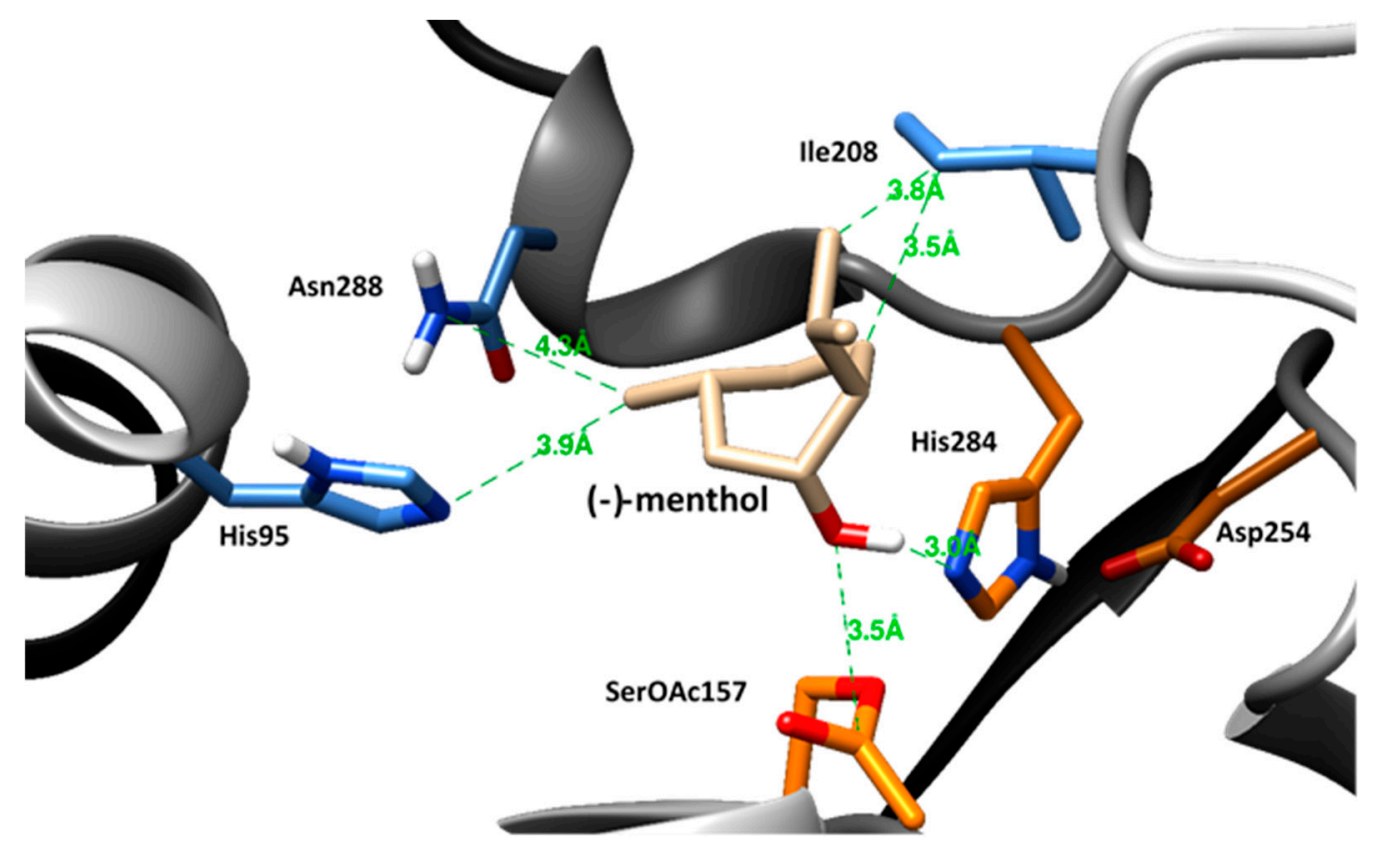

Figure 1. Interaction network for (-)-menthol within PestE (PDB code: 3ZWQ, [24]) based on a substrate docking. Residues interacting with (-)-menthol are shown in light blue, residues of the catalytic triad Ser157, His284, Asp254 are in orange. The model was created based on an acetylated Ser157.

PestE wild type and variants were purified by a two-step approach consisting of affinity chromatography followed by heat precipitation. The purity of the purified enzymes was evaluated by SDS-PAGE (Figure S2). Subsequently, several monoterpenes were used as model compounds to determine the acyltransferase activity of PestE enzymes towards these primary (citronellol-Figure 2a and Table S1), secondary (menthol-Table 1), tertiary (linalool—data not shown), and phenolic (carvacrol-Table S3) alcohols with vinyl acetate as an acyl donor. In all reactions, $0.1 \%(v / v)$ Triton-X-100 was added because previous studies found that Triton-X-100 prevented the protein instability that occurs at low concentrations of purified PestE [22].

The results revealed that only the tertiary alcohol linalool was not converted, despite the efforts to increase the substrate entrance space, possibly due to the high steric hindrance and less reactivity of this tertiary alcohol. Reactions with primary and secondary alcohols showed similar conversions for all variants, although the conversion rate was lower for PestE_N288F. It is also obvious that the variant PestE_N288F decreased the undesired product hydrolysis (Table S1 and Table 1). Regarding the phenolic alcohol carvacrol, low conversions were obtained for all PestE variants, still, this is the first time a promiscuous acyltransferase was reported to acylate a phenolic alcohol in an aqueous environment (Table S3).

Table 1 shows that all investigated variants catalyze the conversion of menthol to menthyl acetate with similar maximum conversions. The results (Table 1) confirmed low enantioselectivity for the wild type and PestE_H95A, and moderate E-values for the PestE_N288F mutant. Contrary to this, PestE_I208A is highly enantioselective for the acetylation of (-)-menthol, showing an enantiomeric excess of $94 \% e e$ and an E-value over 55. Additionally, the initial acylation is higher for PestE_I208A compared to the wild type. These results suggest that the increase of the tunnel size by rational design facilitated the access of (-)-menthol to the enzyme's active site. 
Table 1. Menthyl acetate conversion and product enantiomeric excess from transesterification reactions catalyzed by PestE wild type and mutants.

\begin{tabular}{ccccccccc}
\hline \multirow{2}{*}{$\begin{array}{c}\text { Time } \\
(\boldsymbol{m i n})\end{array}$} & \multicolumn{2}{c}{ PestE_wt } & \multicolumn{2}{c}{ PestE_H95A } & \multicolumn{2}{c}{ PestE_I208A } & \multicolumn{2}{c}{ PestE_N288F } \\
\cline { 2 - 8 } & Conv (\%) & $\% e e(\mathbf{E})$ & Conv (\%) & \%ee (E) & Conv (\%) & \%ee (E) & Conv (\%) & $\% e e$ (E) \\
\hline 10 & 0 & - & 0 & - & 12 & 100 & 0 & - \\
30 & 7 & 74 & 3 & 67 & 17 & 100 & 3 & 100 \\
60 & 18 & 67 & 8 & 81 & 31 & $95(59)$ & 9 & 97 \\
120 & 32 & $59(5)$ & 24 & 66 & 36 & $94(55)$ & 20 & 89 \\
180 & 37 & $58(5)$ & 31 & $62(6)$ & 38 & $94(58)$ & 29 & 81 \\
240 & 38 & $57(5)$ & 28 & 66 & 36 & $95(67)$ & 35 & $79(13)$ \\
480 & 32 & $68(7)$ & 31 & $71(8)$ & 24 & 100 & 44 & $80(17)$ \\
1440 & 19 & 80 & 9 & 67 & 13 & 100 & 38 & $79(14)$ \\
\hline
\end{tabular}

Conversion (\%) and optical purity (\%ee) were determined from GC-MS analytical data using a chiral column; The E-value was determined according to Rakels, Straathof, and Heijnen [29]. Reaction conditions: molar ratio 1:10 (( \pm$)$-menthol:vinyl acetate), using $20 \mathrm{mM}( \pm)$-menthol, $0.1 \%(v / v)$ Triton-X-100, $0.2 \mu \mathrm{g} \cdot \mathrm{mL}-1 \mathrm{of}$ the purified enzymes in $1 \mathrm{~mL}$ of an aqueous buffer $(50 \mathrm{mM}$ potassium phosphate, $300 \mathrm{mM}$ sodium chloride, $\mathrm{pH} 8.0)$ at 1000 $\mathrm{rpm}$ and $40{ }^{\circ} \mathrm{C}$. Reactions were conducted in triplicate, and the average values are shown. Reactions without enzymes did not result in ester products.

Transesterification reactions with ethyl acetate, a much less activated acyl donor compared to vinyl acetate, are reversible and, hence, could lead to lower conversion to esterified products. As all the PestE variants were shown to be efficient biocatalysts for the acylation of citronellol, this substrate was used in reactions with ethyl acetate. The results, shown in Figure $2 \mathrm{~b}$ and Table S2, demonstrate that all PestE variants studied can perform the acylation with ethyl acetate as an acyl donor almost equally as well as with vinyl acetate. The PestE_N288F variant enabled higher conversion $(92 \%$ ester formed within $4 \mathrm{~h}$ ) in comparison to the other acyltransferase variants, where a maximum of $83 \%$ was observed together with subsequent hydrolysis of the ester product (Table S2). This finding could be linked to a potential reorganization of the water network (Figures S3-S5). Thus, the undesired and commonly observed hydrolysis of ester products was slowed down, which can be explained by its disrupted water network (Figure S5), decreasing the hydrolytic activity.

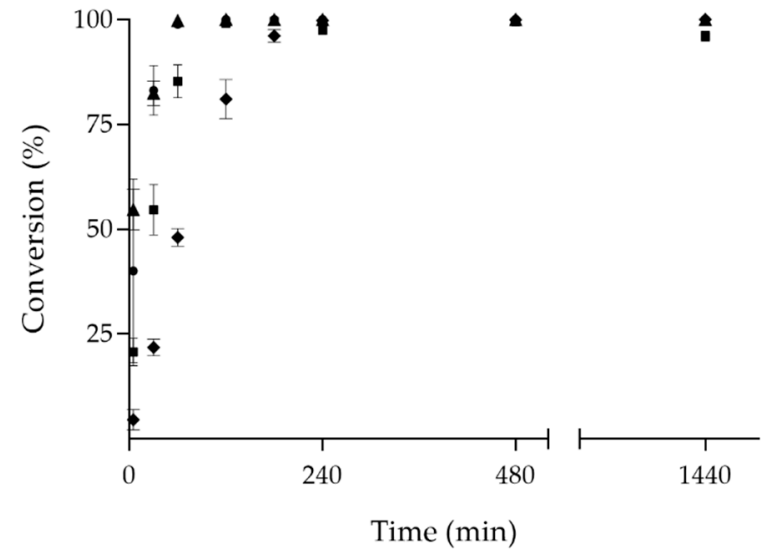

(a)

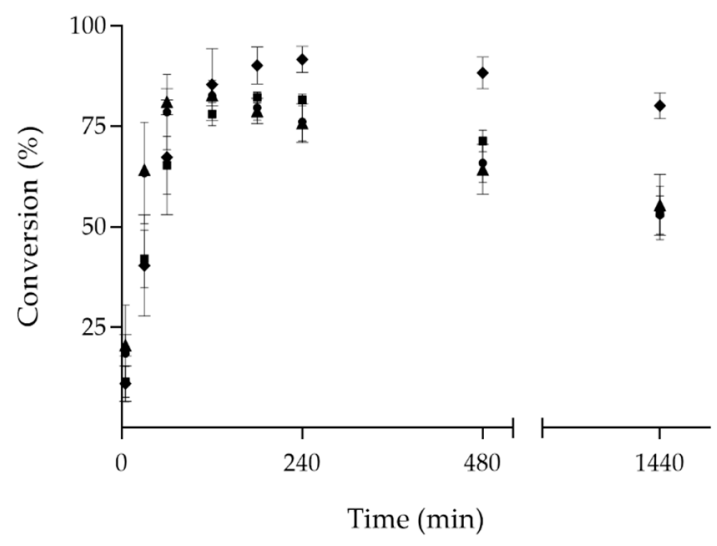

(b)

Figure 2. Enzymatic acylation of $( \pm)$-citronellol over time with (a) vinyl acetate and (b) ethyl acetate as acyl donors. Where:

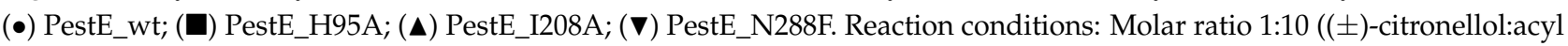
donor), using $20 \mathrm{mM}( \pm)$-citronellol, $0.1 \%(v / v)$ Triton-X-100, $20 \mu \mathrm{g} \cdot \mathrm{mL}^{-1}$ of the purified enzymes when ethyl acetate was used, or $0.2 \mu \mathrm{g} \cdot \mathrm{mL}^{-1}$ of the purified enzymes when vinyl acetate was used, in $1 \mathrm{~mL}$ of an aqueous buffer ( $50 \mathrm{mM}$ potassium phosphate, $300 \mathrm{mM}$ sodium chloride, $\mathrm{pH}$ 8.0) at $1000 \mathrm{rpm}$ and $40^{\circ} \mathrm{C}$. Reactions were conducted in triplicate, and the average values are shown. Reactions without enzymes did not result in ester products. 


\section{Discussion}

In this study, we performed rational protein engineering of the Pyrobaculum calidifontis VA1 esterase (PestE) using two different concepts, the first to increase the hydrophobicity of the active site, with the mutations H95A and N288F, aiming to disrupt the water network and to increase affinity to organic nucleophiles. The second approach was to increase the tunnel size by the mutation I208A. Interestingly, the increased hydrophobicity with the mutations H95A and N288F did not increase the acyltransferase activity, although it is believed that increased hydrophobicity facilitates binding of (non-polar) acyl acceptors $[5,15,30]$. However, the undesired subsequent hydrolysis of the acylated product was substantially reduced by the variant PestE_N288F, which showed very high up to complete conversions to the ester products and reduced subsequent hydrolysis. The reduced hydrolysis could be due to inactivation of the water molecules in the active site by the introduction of phenylalanine (Figure S5). In the same way, the variant PestE_H95A showed higher hydrolysis as the water network was strengthened (Figure S4), although the hydrophobicity increased. Thus, the reorganized water network in the active site seems to affect hydrolysis activity stronger than the increase in hydrophobicity. For completeness, it has to be mentioned that the PestE_N288F variant presented higher electrophoretic mobility (Figure S2), which might be caused by protein digestion; nevertheless, this variant still showed very high activity. Meanwhile, the variant I208A is highly selective on the (-)-menthol acylation, which can be related to a better access or a better binding of the substrate into the enzyme active site. Again, the decreased hydrophobicity did not decrease but increased the acyltransferase activity in the mutant PestE_I208A, emphasizing that factors such as the access to the binding site are, in this particular case, more relevant for the conversion of monoterpene substrates. Consequently, the highest conversion of the challenging substrate carvacrol was achieved with PestE_I208A, demonstrating not only acyltransferase activity on phenolic alcohols for the first time, but also that enzyme activity is limited by access to the active site. In the same manner, the conversion of tertiary alcohols is eventually only limited by the accessibility of the active site. These findings might help to develop effective acyltransferases for further applications. The outstanding enzymatic activities of the PestE variants for good substrates as citronellol underline the synthetic potential of acyltransferase-catalyzed acylation reactions in aqueous solutions. Even though the protocol presented in this paper requires the addition of Triton-X-100 for the protein stabilization [22], in experiments with heat shock-enriched lysate (without affinity chromatography purification, data not shown) this was not necessary, which can facilitate the enzyme application. Furthermore, we could show that the acyl donor vinyl acetate can be replaced by the less toxic and more environmentally friendly ethyl acetate, in relation to vinyl acetate [31], still leading to high conversions at relatively short reaction times.

\section{Conclusions}

In this study, novel PestE variants have been designed for the acylation of monoterpene in water. All variants represented high activity for citronellol. PestE_I208A presented high enantioselectivity for the acetylation of (-)-menthol. PestE_N288F showed good acyl transfer in water, reducing the commonly observed hydrolysis of the monoterpene esters formed upon prolonged reaction times. This variant also catalyzes acylation successfully with the cheaper and environmentally friendly acyl donor ethyl acetate. With carvacrol, a phenolic alcohol was acylated with a promiscuous acyltransferase for the first time.

Supplementary Materials: The following are available online at https:/ /www.mdpi.com/article/10 .3390 / microorganisms9081790/s1, Figure S1: Enzymatic acylation of $( \pm)$-menthol over time with vinyl acetate as acyl donor, Figure S2: SDS-PAGE analysis of samples of the purified PestE (wt and variants) used in this study, Table S1: Conversion (\%) of ( \pm )-citronellol and vinyl acetate to citronellyl acetate over time using PestE wild type and mutants as biocatalysts, Table S2: Conversion (\%) of $( \pm)$-citronellol and ethyl acetate to citronellyl acetate over time using PestE wild type and mutants as biocatalysts, Table S3: Conversion (\%) of carvacrol to carvacryl acetate over time using PestE wild 
type and mutants as biocatalysts, Figure S3: Water network of PestE wild type active site, Figure S4: Water network of PestE_H95A active site, Figure S5: Water network of PestE_N288F active site.

Author Contributions: U.T.B. and A.S. conceived the project; A.S. and H.T. equally designed the methodology and performed the investigation; J.K. performed the investigation; H.M. executed formal analysis; S.P.G. contributed to the methodology; I.I.J., I.C.R.L., and U.T.B. accomplished the funding; U.T.B. administered and supervised the project; A.S. and H.T. wrote the original draft that all authors contributed to. All authors have read and agreed to the published version of the manuscript.

Funding: A.S. received funding from the Coordenação de Aperfeiçoamento de Pessoal de Nível Superior-Brasil (Capes)_Finance Code 001. H.T. was funded by the Leibniz Association's strategic networking funding program Leibniz ScienceCampus ComBioCat.

Institutional Review Board Statement: Not applicable.

Informed Consent Statement: Not applicable.

Data Availability Statement: Data are contained within the article and the supplementary material.

Acknowledgments: The authors are grateful to Haruyuki Atomi, Kyoto University (Kyoto, Japan) for providing the gene encoding PestE.

Conflicts of Interest: The authors declare no conflict of interest. Additionally, the funders had no role in the design of the study; in the collection, analyses, or interpretation of data; in the writing of the manuscript, or in the decision to publish the results.

\section{References}

1. Bornscheuer, U.T.; Kazlauskas, R.J. Hydrolases in Organic Synthesis, 2nd ed.; Wiley-VCH: Weinheim, Germany, 2006.

2. Henke, E.; Pleiss, J.; Bornscheuer, U.T. Activity of lipases and esterases towards tertiary alcohols: Insights into structure- function relationships. Angew. Chem. Int. Ed. 2002, 41, 3211-3213. [CrossRef]

3. Liese, A.; Seelbach, K.; Wandrey, C. (Eds.) Industrial Biotransformations, 2nd ed.; Wiley-VCH: Weinheim, Germany, 2006.

4. Bornscheuer, U.T.; Kazlauskas, R.J. Catalytic promiscuity in biocatalysis: Using old enzymes to form new bonds and follow new pathways. Angew. Chem. Int. Ed. 2004, 43, 6032-6040. [CrossRef]

5. Müller, H.; Becker, A.-K.; Palm, G.J.; Berndt, L.; Badenhorst, C.P.S.; Godehard, S.P.; Reisky, L.; Lammers, M.; Bornscheuer, U.T. Sequence-based prediction of promiscuous acyltransferase activity in hydrolases. Angew. Chem. Int. Ed. 2020, 59, 11607-11612. [CrossRef]

6. Krishna, S.H.; Persson, M.; Bornscheuer, U.T. Enantioselective transesterification of a tertiary alcohol by lipase A from Candida antarctica. Tetrahedron Asymmetry 2002, 13, 2693-2696. [CrossRef]

7. Wikmark, Y.; Humble, M.S.; Bäckvall, J.-E. Combinatorial library based engineering of Candida antarctica lipase A for enantioselective transacylation of sec-alcohols in organic solvent. Angew. Chem. Int. Ed. 2015, 54, 4284-4288. [CrossRef] [PubMed]

8. Herbst, D.; Peper, S.; Niemeyer, B. Enzyme catalysis in organic solvents: Influence of water content, solvent composition and temperature on Candida rugosa lipase catalyzed transesterification. J. Biotechnol. 2012, 162, 398-403. [CrossRef] [PubMed]

9. Holmberg, E.; Szmulik, P.; Norin, T.; Hult, K. Hydrolysis and esterification with lipase from Candida cylindracea. Influence of the reaction conditions and acid moiety on the enantiomeric excess. Biocatalysis 1989, 2, 217-223. [CrossRef]

10. Wu, X.Y.; Jääskeläinen, S.; Linko, Y.-Y. An investigation of crude lipases for hydrolysis, esterification and transesterification. Enzym. Microb. Technol. 1996, 19, 226-231. [CrossRef]

11. van Rantwijk, F.; Hacking, M.A.P.J.; Sheldon, R.A. Lipase-catalyzed synthesis of carboxylic amides: Nitrogen nucleophiles as acyl acceptor. Monatsh. Chem. 2000, 131, 549-569. [CrossRef]

12. Yang, Z.; Huang, Z.-L. Enzymatic synthesis of sugar fatty acid esters in ionic liquids. Catal. Sci. Technol. 2012, 2, 1767-1775. [CrossRef]

13. Godehard, S.P.; Badenhorst, C.P.S.; Müller, H.; Bornscheuer, U.T. Protein engineering for enhanced acyltransferase activity, substrate scope, and selectivity of the Mycobacterium smegmatis acyltransferase MsAcT. ACS Catal. 2020, 10, 7552-7562. [CrossRef]

14. Neungot, V.; Moulin, G.; Dubreucq, E.; Bigey, F. The lipase/acyltransferase from Candida prapsilosis—Molecular cloning and characterization of purified recombinant enzymes. Eur. J. Biochem. 2002, 269, 1734-1745.

15. Mathews, I.; Soltis, M.; Saldajeno, M.; Ganshaw, G.; Sala, R.; Weyler, W.; Cervin, M.A.; Whited, G.; Bott, R. Structure of a novel enzyme that catalyzes acyl transfer to alcohols in aqueous conditions. Biochemistry 2007, 46, 8969-8979. [CrossRef] [PubMed]

16. Godehard, S.P.; Müller, H.; Badenhorst, C.P.S.; Stanetty, C.; Suster, C.; Mihovilovic, M.D.; Bornscheuer, U.T. Efficient acylation of sugars and oligosaccharides in aqueous environment using engineered acyltransferases. ACS Catal. 2021, 11, 2831-2836. [CrossRef]

17. Müller, H.; Godehard, S.P.; Palm, G.J.; Berndt, L.; Badenhorst, C.P.S.; Becker, A.-K.; Lammers, M.; Bornscheuer, U.T. Discovery and design of family VIII carboxylesterases as highly efficient acyltransferases. Angew. Chem. Int. Ed. 2021, 60, 2013-2017. [CrossRef] 
18. Reisky, L.; Srinivasamurthy, V.S.T.; Badenhorst, C.P.S.; Godehard, S.P.; Bornscheuer, U.T. A novel high-throughput assay enables the direct identification of acyltransferases. Catalysts 2019, 9, 64. [CrossRef]

19. Zeng, S.; Liu, J.; Anankanbil, S.; Chen, M.; Guo, Z.; Adams, J.P.; Snajdrova, R.; Li, Z. Amide synthesis via aminolysis of ester or acid with an intracellular lipase. ACS Catal. 2018, 8, 8856-8865. [CrossRef]

20. Müller, J.; Sowa, M.A.; Dörr, M.; Bornscheuer, U.T. The acyltransferase activity of lipase CAL-A allows efficient fatty acid esters formation from plant oil even in an aqueous environment. Eur. J. Lipid Sci. Technol. 2015, 117, 1903-1907. [CrossRef]

21. Land, H.; Hendil-Forssell, P.; Martinelle, M.; Berglund, P. One-pot biocatalytic amine transaminase/acyl transferase cascade for aqueous formation of amides from aldehydes or ketones. Catal. Sci. Technol. 2016, 6, 2897-2900. [CrossRef]

22. Hotta, Y.; Ezaki, S.; Atomi, H.; Imanaka, T. Extremely stable and versatile carboxylesterase from a hyperthermophilic archaeon. Appl. Environ. Microbiol. 2002, 68, 3925-3931. [CrossRef]

23. Kourist, R.; de María, P.D.; Bornscheuer, U.T. Enzymatic synthesis of optically active tertiary alcohols: Expanding the biocatalysis toolbox. ChemBioChem 2008, 9, 491-498. [CrossRef] [PubMed]

24. Palm, G.J.; Fernandez-Álvaro, E.; Bogdanović, X.; Bartsch, S.; Sczodrok, J.; Singh, R.K.; Böttcher, D.; Atomi, H.; Bornscheuer, U.T.; Hinrichs, W. The crystal structure of an esterase from the hyperthermophilic microorganism Pyrobaculum calidifontis VA1 explains its enantioselectivity. Appl. Environ. Microbiol. 2011, 91, 1061-1072. [CrossRef] [PubMed]

25. Krieger, E.; Darden, T.; Nabuurs, S.B.; Finkelstein, A.; Wriend, G. Making optimal use of empirical energy functions: Force-field parameterization in crystal space. Proteins 2004, 57, 678-683. [CrossRef]

26. Pettersen, E.F.; Goddard, T.D.; Huang, C.C.; Couch, G.S.; Greenblatt, D.M.; Meng, E.C.; Ferrin, T.E. UCSF chimera-A visualization system for exploratory research and analysis. J. Comput. Chem. 2004, 25, 1605-1612. [CrossRef]

27. Trott, O.; Olson, A.J. AutoDock Vina: Improving the speed and accuracy of docking with a new scoring function, efficient optimization and multithreading. J. Comput. Chem. 2010, 31, 455-461. [CrossRef]

28. Kokkonen, P.; Bednar, D.; Pinto, G.; Prokop, Z.; Damborsky, J. Engineering enzymes access tunnels. Biotechnol. Adv. 2019, 37, 107386. [CrossRef]

29. Rakels, J.L.L.; Straathof, A.J.J.; Heijnen, J.J. A simple method to determine the enantiomeric ratio in enantioselective biocatalysis. Enzym. Microb. Technol. 1993, 15, 1051-1056. [CrossRef]

30. Kazemi, M.; Sheng, X.; Kroutil, W.; Himo, F. Computational study of Mycobacterium smegmatis acyl transferase reaction mechanism and specificity. ACS Catal. 2018, 8, 10698-10706. [CrossRef]

31. Paravidino, M.; Hanefeld, U. Enzymatic acylation: Assessing the greenness of different acyl donors. Green Chem. 2011, 13, 2651-2657. [CrossRef] 